\title{
A fractional order impedance model to capture the structural changes in lungs
}

\author{
Clara M. Ionescu* Dana Copot* Robin De Keyser* \\ * Ghent University, Faculty of Engineering and Architecture, \\ Department of Electrical energy, Systems and Automation, \\ Technologiepark 913, B9052 Zwijnaarde, Belgium (e-mail: \\ \{ClaraMihaela.Ionescu, Dana.Copot, Robain.DeKeyser\} @UGent.be).
}

\begin{abstract}
This paper introduces a correlation analysis between the structural changes occurring in the lungs and the corresponding variations in the fractional order value of an impedance model. It was discussed how variations in the wall thickness, cross-sectional area and elastic moduli in distal airways involved in respiratory process affect changes in the fractional order value. Two lumped models were discussed: i) a theoretical model derived from morphological information and ii) a lumped parametric model. Results indicate that a correlation analysis is possible for various degrees of obstruction and effects may be directly related to the identified fractional order value.
\end{abstract}

Keywords: mechanical properties, recursive, frequency response, adaptation, biology, respiratory system, fractional order impedance.

\section{INTRODUCTION}

The emerging concepts of fractional calculus (FC) in biology and medicine have shown a great deal of success, explaining complex phenomena with a startling simplicity (West et al. (1986); Magin (2006)). It is clear that a major contribution of the concept of FC has been and remains still in the field of biology and medicine (Machado et al. (2013)). Fractional calculus generously allows integrals and derivatives to have any order, hence the generalization of the term fractional-order to that of general-order. Of all applications in biology, linear viscoelasticity is certainly the most popular field, for their ability to model hereditary phenomena with long memory (Berg (2009)). Viscoelasticity has been shown to be the origin of the appearance of FO models in polymers (from the Greek: poly - many and meros - parts) (Adolfsson et al. (2005)) and resembling biological tissues (Doehring et al. (2005); Suki et al. (1994)).

Viscoelasticity of the lungs is characterized by compliance, expressed as the volume increase in the lungs for each unit increase in alveolar pressure or for each unit decrease of pleural pressure. The most common representation of the compliance is given by the pressure-volume (PV) loops. Changes in elastic recoil (more, or less, stiffness) will affect these pressure-volume relationships. These changes are fueled by structural variations during the progress of age, or pathology, or both. In clinical terms, this is known as airway remodeling.

The term airway remodeling refers to the process of modification and sustained disruption of structural cells and tissues leading to a new airway-wall structure with

\footnotetext{
* This research is supported by the Flemish Research Council (FWO), Research Project FWOPR2013 005101. C.M. Ionescu is a post-doctoral fellow of FWO.
}

implicit new functions. Airway remodeling is supposed to be a consequence of long-term airway diseases. Some studies suggest that the remodeling may be a part of the primary pathology rather than simply a result of chronic inflammation (Berg (2009)). Of crucial importance in this quest to understand airway remodeling is the composition and structure of the lung tissue (Tgavalekos et al. (2003); Kaczka and Dellaca (2011)). The composition and structure determines the mechanical properties of the lungs. Structural changes will induce alternations in tissue elasticity and viscosity.

In this paper we aim to determine a correlation between the structural changes occurring in the lungs and variation in the fractional order value of an impedance model (FOIM). The next section will provide a brief clinical perspective of airway remodeling. The third section presents the electrical analogy to airway models and discusses the effect on the model parameters due to the changes in morphology. The fourth section presents the simulation study of these changes and their effects on the fractional order value. A conclusion section summarizes the main outcome of this work.

\section{STRUCTURAL CHANGES IN THE LUNGS WITH DISEASE}

If a failure in the nominal operation and function occurs in the lungs, the adaptation mechanism will be triggered in an attempt to ensure species survival. This implies ensuring a minimum of vital capacity, which is a balance between changes in alveolar pressure and lung volume during the breathing process. As a defensive mechanism to external agents, the airways and the parenchymal tissue may undergo inflammation, constriction, fibrosis, etc. Structural alternations introduced by pathological processes are traditionally divided into three layers: the inner 
wall, the outer wall and the smooth-muscle layer. The inner wall exist of the epithelium, basement membrane and submucosa, while the outer layer consists of cartilage and loose connective tissue between the muscle layer and the surrounding lung parenchyma.

In COPD (chronic obstructive pulmonary disease), major structural alternations occur in the small bronchi and membranous bronchiole (airway diameter $<2 \mathrm{~mm}$ ). Changes occur around the supporting cartilage and bronchial glands in the peripheral airways $(\approx 2 \mathrm{~mm}$ diameter). Here, the thickening occurs mainly in the inner wall area of the large airways (Lande and Mitzner (2006); Berg (2009); Kaczka and Dellaca (2011)).

The most important changes in asthma are located in the conducting airways (i.e. levels 1-16), which can thicken up to $300 \%$. Asthma patients have thickened segmental and subsegmental bronchial walls over their entire size rage. This thickening is dependent on the degree of the disease, more severe and older patients will depict these characteristics more than young patients (Berg (2009); Lai and Hyatt (2000)). In asthma, the inflammatory reactions takes place in the higher part of the airways than in COPD. Unfortunately for COPD patients, the airway obstruction that accompanies these changes is resistant to medication which makes the changes persistent. By contrast, in asthma the inflammatory processes can be controlled by the use of corticosteroids. There are also important differences in the remodeling of the extracellular matrix and the role of proteolytic enzymes and growth factors which lead to specific airway remodeling results by disease. More clinical information about inflammation mechanics in airway remodeling can be found in (Berg (2002)). For remodeling effects in asthma, an important role is played by the degree to which the smooth muscle surrounds the airway lumen.

Mucous, produced in both asthma as COPD, is quantitatively and qualitatively abnormal with alternations in its molecular and cellular composition (Hogg et al. (2004)). The elevated ratio of mucous/serous acini provides a secretion of a thicker, gel-like mucus in COPD. Partial or complete occlusion of the small airways occurs.

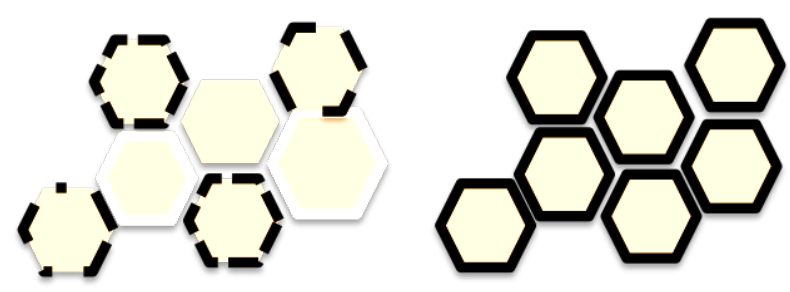

Fig. 1. Schematic representation of the honeycomb-like alveolar structure. Right: healthy structure. Left: broken walls.

The lungs consist of large surface areas with small diffusion distances to guarantee proper gas exchange. The 3D structure of the alveoli can be compared to a honeycomb structure of thin-walled septa which form a fractal network (Hogg et al. (2004); Ionescu et al. (2009a)) as in figure 1. Changes in mechanical properties of the lung tissue by pathology will influence this process. Once an alveolar wall starts to rupture in COPD, the stress the original wall carried is redistributed to the neighboring walls. If this stress is high, a single rupture will induce a cascade of ruptures and serves as a positive feedback for further breakdown. It is obvious that there is a point beyond which the structure-function relationship cannot return to the healthy condition. It is therefore useful to correlate these changes with model parameters for analysis.

\section{ANALYSIS}

By analogy to electrical networks, one may consider voltage as the equivalent for respiratory pressure $P$ and current as the equivalent for air-flow $Q$ (Kundur (1994)). Electrical resistances $R_{e}$ may be used to represent respiratory resistance that occur as a result of air-flow friction in the airways. Similarly, electrical capacitors $C_{e}$ may represent volume compliance of the airways which allows them to inflate/deflate. We will discuss in this section their definitions as a function of morphology and their role within the airway remodeling process.

\subsection{Parameters}

In (Ionescu et al. (2009b)) was developed the electrical analogy to transmission lines for the mechanical parameters in the elastic and viscoelastic airways. The model was based on the geometrical parameters: radius and wall thickness $(R, h)$, on the mechanical characteristics of the airway tube: complex elastic moduli (given by its modulus and angle) and Poisson coefficient $\left(|E|, \varphi_{E}, \nu_{P}\right)$, and on the air properties: viscosity and density $(\mu, \rho)$. Over the length $\ell$ of an airway tube, we have the corresponding definitions for resistance:

$$
R_{e}=\ell \frac{\mu \delta^{2}}{\pi R^{4} M_{1}} \sin \left(\varepsilon_{1}\right),
$$

for inductance:

$$
L_{e}=\ell \frac{\rho}{\pi R^{2}} \frac{\cos \left(\varepsilon_{1}\right)}{M_{1}},
$$

for conductance:

$$
G_{e}=\ell \omega \frac{2 \pi R^{3}\left(1-\nu_{P}^{2}\right)^{2}}{|E| h} \sin \varphi_{E},
$$

and for capacitance (compliance)

$$
C_{e}=\ell \frac{2 \pi R^{3}\left(1-\nu_{P}^{2}\right)^{2}}{|E| h} \cos \varphi_{E},
$$

where $x$ is the longitudinal coordinate, $M_{1}$ is the modulus of the Bessel function of order 1 , and $\omega$ is the angular frequency.

Since in pathology, viscoelastic effects are most pronounced at low frequencies, we can neglect inductance and assume lossless airways (i.e. neglect the heat production from molecular friction).

The values for airway radius, thickness, elastic moduli, etc have been used as those published in (Ionescu (2013)). The resistance and compliance per branch are depicted in figure 2. The consecutive ratios between these branches for resistance and compliance are given in figure 3 . It can be observed that the ratios of resistance are above 1 , indicating an increase with every level - this is obvious, 

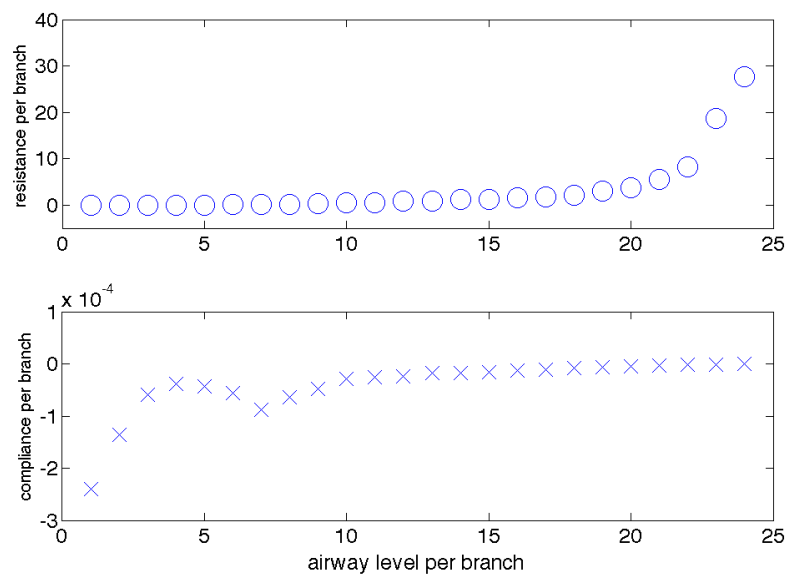

Fig. 2. Evolution of resistance $\left(\mathrm{kPa}^{*} \mathrm{~s} / \mathrm{L}\right)$ and compliance $(\mathrm{L} / \mathrm{kPa})$ values per branch in normal airways.

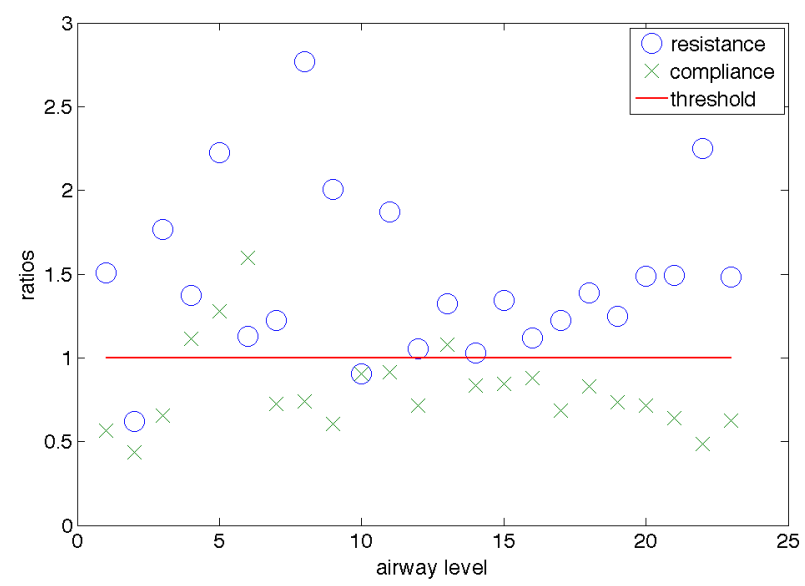

Fig. 3. Evolution of the ratios in normal airways with consecutive branches in consecutive airway levels.

since we are looking at each branch solely, and the crosssectional area is diminishing. The compliance is below 1 , indicating that it increases, as a result of the elastic moduli, with more soft tissue and less cartilage percentage at lower branches in the respiratory tree. This is indeed the case, since these lower airways are those which need to expand during inspiration (i.e. alveolar levels where diffusion occurs).

\subsection{Changes in airway radius, wall thickness and elastic modulus}

Here we investigate the effect of changes in the airway radius, airway wall thickness and elastic modulus of the tissue. These changes are significant for obstructive disease such as asthma and COPD. However, the most important are the irreversible changes in COPD, since these will always mark the respiratory impedance and be visible in all lung function tests. Changes in the respiratory zone (i.e. airways below level 16) in the distal airways and lung parenchyma.

Firstly, let us look at changes in the airway thickness. This may occur since the lung begins to 'protect' itself from the

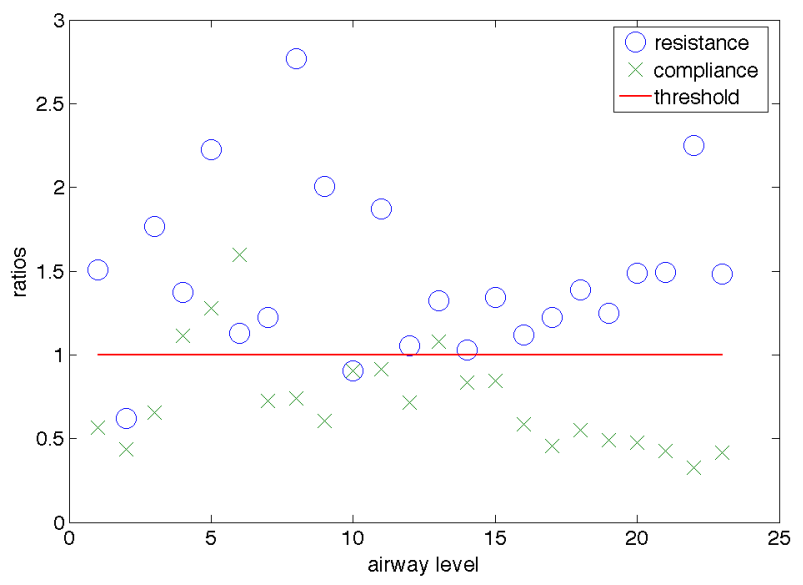

Fig. 4. Evolution of the ratios in thickened airways with consecutive branches in consecutive airway levels.

damaged cells in the airway soft tissue. In time, fibrosis occurs and thickness may increase significantly. Figure 4 depicts the variation in the resistance and compliance ratios per branch with each airway level in case of increased changes in thickness with $50 \%$.

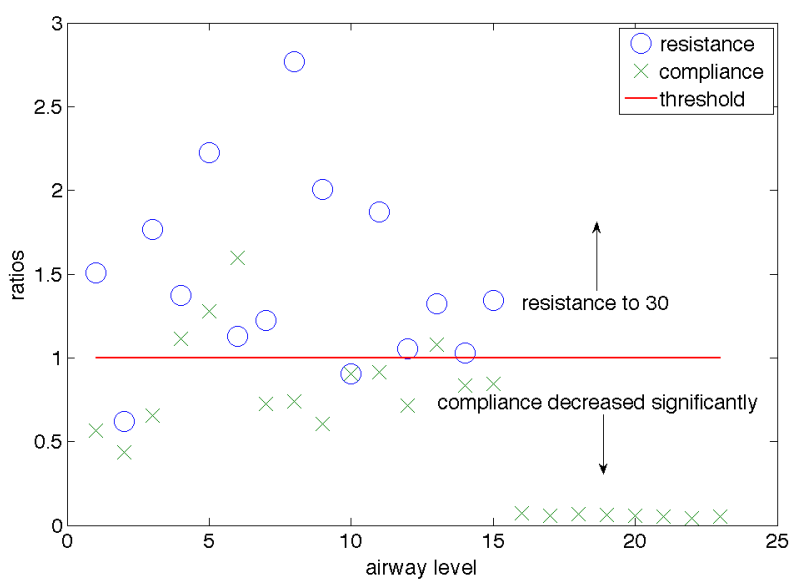

Fig. 5. Evolution of the ratios in thickened and obstructed airways with consecutive branches in consecutive airway levels.

Secondly, the change in thickness usually induces a change in the radius. This become obstructed and mucus may result from the inflammation of the airway soft tissue. Mucus secretion may obdurate totally parts of the airway, producing exacerbations, hence the patient will cough with sputum secretions. Figure 5 depicts the variation in the resistance and compliance ratios per branch with each airway level in case of decreasing the radius with $50 \%$.

Finally, the changes in thickness and radius are usually resulting in a change in the elastic modulus of the soft tissue and the cartilage tissue in the airways. Typically, fibrosis will reduce the overall elasticity of the modulus. Figure 6 depicts the variation in the resistance and compliance ratios per branch with each airway level in case of stiffening the soft and cartilage tissue with $30 \%$. 


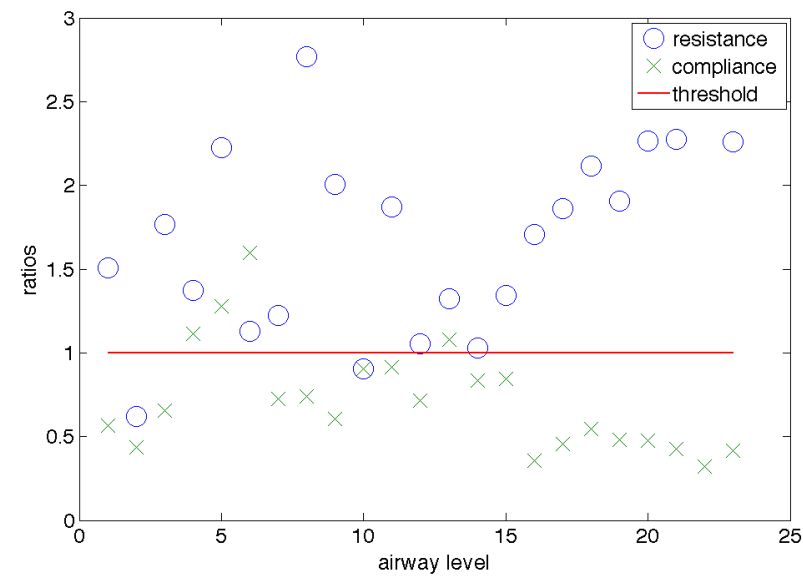

Fig. 6. Evolution of the ratios in fibrotic airways with consecutive branches in consecutive airway levels.

It is obvious that the changes with most impact are those related to obstruction. Fibrosis, a slow process, will also contribute to the increase in resistance and thus will impede the air passage through the airways. The wall ruptures discussed in figure 1 will eventually decrease the resistance because the air will be able to pass more easily (Hogg et al. (2004)). However, since the elastic structure is lost, the tissue will not be able to inflate/deflate as normal and the air may remain entrapped, causing the 'barrel chest', a process visible in COPD patients. The shallow breathing of COPD patient indicates that the air goes easily into the lungs, but it is not easily expired, requiring an increase if the work of breathing, resulting in fatigue and pulmonary stress. If the honeycomb structure becomes damaged over a significant area, these alveolar structures are no longer prompted in the breathing process, and parts of the lungs may lose function. As the disease progresses, the COPD patient requires additional mechanical ventilation and oxygen supply.

\section{RELATION TO FOIMS}

The respiratory tract can be approximated well by its electrical analogue, where current denotes flow changes and voltage denotes pressure changes. If the morphological structure of the lungs is preserved, then the quasi-fractal structure of the lungs may be employed, simplifying significantly the mathematical burden of the model (Ionescu et al. (2009b)). It has been shown in (Oustaloup (1995); Ionescu (2013)) that such a quasi-fractal structure, i.e. a ladder network with recurrent impedance elements of the form:

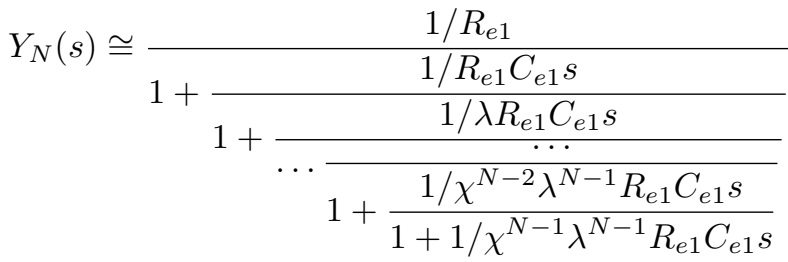

with $R_{e 1}$ and $C_{e 1}$ denoting the resistance and compliance in the first airway (i.e. trachea), and $\lambda$ the ratio of resistances per total levels, $\chi$ the ratio of compliances per total levels and $s$ the Laplace operator, can be well- approximated for $N \rightarrow \infty$ airway levels by the compact form of admittance:

$$
Y_{N}(s) \cong \frac{1 / R_{e 1}}{K(\lambda, \chi) \cdot\left(1 / R_{e 1} C_{e 1} s\right)^{n}}
$$

with $K(\lambda, \chi)$ a gain factor depending on the values of the ratios and the fractional order $n$ given by

$$
n=\frac{\log (\lambda)}{\log (\lambda)+\log (\chi)}
$$

Notice that the impedance, $Z_{N}(s)$ will be the inverse of the admittance. In the frequency domain, the fractional order will lead to a constant-phase behavior, i.e. a phaselocking in the frequency range given by the convergence conditions (Oustaloup (1995); Ionescu (2013)). Depending on the number $N$ of cells in the ladder network, the constant-phase behavior will emerge over a wider range of frequencies. This result is applicable to any kind of ladder network (airways, arteries, etc). However, the fractional order value and coefficients will change according to the properties (morphology, geometry) of the system. It has also been shown in (Ionescu (2013)) that the convergence is not necessary for $N \rightarrow \infty$; after a limited number of cells in the ladder network, e.g. $N=7$ one may already observe the constant-phase behavior obeying relation (7).

For the changes discussed in the previous section, one may calculate the corresponding variations in the fractional order value $n$. Figure 7 shows the variations occurring in the magnitude and phase of the impedance from (5) with increasing values in the ratios of the resistance. As the ratio $\lambda$ increases, so is the value of $n$, changing the phase lock value and the slope of the magnitude. The values used to calculate this figure are nominal ones as reported in (Ionescu (2013)), well correlated to real life values; however, the trends for frequency approaching $-\infty$ are of course overestimated. The constant-phase specific for FOIMs is pronounced in the frequencies above $10^{-2}$. For real values of the elements in (5) we refer the reader to (Ionescu et al. (2013)).
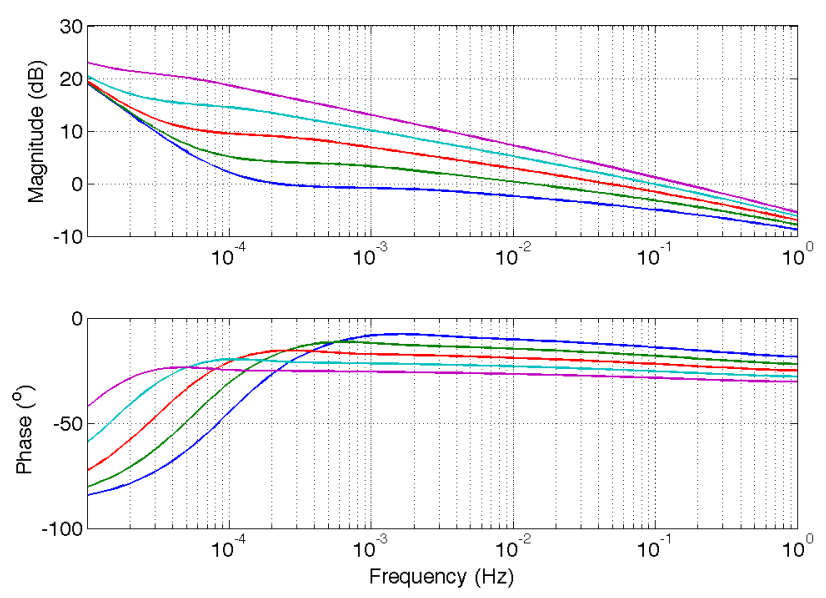

Fig. 7. Evolution of the impedance with increasing values of resistance ratios.

The effect of decreasing compliance (i.e. increasing stiffness) will be opposite to that of increasing resistance. However, it is the relative degree of changes between the 
two which dictates the increase or decrease in $n$ with the progress of the respiratory disease.

\section{RELATION TO VISCOELASTICITY}

To model the combined effects of viscoelastic materials, one may use the relaxation function and use Boltzmann superposition integrals. However, it has been shown by several studies that the relaxation function is not well described by an exponential law, but rather by a powerlaw trend as in (Bates $(2007)) \frac{C(\beta)}{\Gamma(1-\beta) t^{-\beta}}$ with $\Gamma$ the Euler gamma function, and the parameters in $\beta$ are material dependent. This is also supported by other mathematical studies on the pressure and volume changes in the lungs (Suki et al. (1994)). We obtain the following relation:

$$
\sigma(t)=C(\beta)\left(D_{0+}^{\beta} \epsilon\right)(t)
$$

which is generically known as the spring-pot and where $\left(D_{0+}^{\beta} \epsilon\right)$ is the Caputo fractional derivative of order $\beta$ and strain $\epsilon$ with respect to time, defined as (Machado et al. (2013)):

$$
\left(D_{0+}^{\beta} \epsilon\right)(t)=\frac{1}{\Gamma(1-\beta)} \int_{0}^{t}(t-\tau)^{-\beta} \epsilon(\tau) d \tau
$$

The spring-pot element has been introduced for biology applications in (Hantos et al. (1992b,a); Craiem and Armentano (2007)) and the $\beta$ parameter is defined in the range $[0,1]$, with 0 corresponding to pure elastic behavior and 1 corresponding to pure viscous behavior. Biologic systems or tissues with predominant elastic properties are known as elasto-viscous materials and can be characterized by $0<\beta<0.5$, whereas those with predominant viscous properties are visco-elastic, with $0.5<\beta<1$.

Specific for the lungs, the stress relaxation can be written as a function of pressure, decreasing with the logarithm of time following a sudden increase in volume (Berg (2009)):

$$
P(t)=P_{0} t^{-k}=P_{0}\left(\exp ^{\ln (t)}\right)^{-k}
$$

with $k$ a constant. This is the response of the lung to a step change in volume, but can also represent the impulse response in flow. Taking the Fourier transform, one obtains:

$$
\begin{aligned}
& Z(\omega)=F\left\{P_{0} t^{-k}\right\}= \\
& \left(P_{0} \sqrt{\frac{2}{\pi}}\right) \Gamma(1-k)\left\{\frac{\cos \left[(1-k) \frac{\pi}{2}\right]-j \sin \left[(1-k) \frac{\pi}{2}\right]}{\omega^{1-k}}\right\}
\end{aligned}
$$

where

$$
\begin{aligned}
& G=\left(P_{0} \sqrt{\frac{2}{\pi}}\right) \Gamma(1-k) \cos \left[(1-k) \frac{\pi}{2}\right] \\
& H=\left(P_{0} \sqrt{\frac{2}{\pi}}\right) \Gamma(1-k) \sin \left[(1-k) \frac{\pi}{2}\right]
\end{aligned}
$$

and if we define $\beta=1-k$ then we have that

$$
\beta=\frac{2}{\pi} \tan ^{-1} \frac{H}{G}
$$

Lumped FO models have been presented in the literature, under the form:

$$
Z_{F O}(s)=R_{r}+\frac{1}{C_{r} s^{\beta_{r}}}
$$

where the term in $C_{r}$ is the most significant with lower frequency and characterizes viscoelasticity through the following relations. From the identified model parameters one can derive the tissue damping $G_{r}$ and elastance $H_{r}$, defined as (Suki et al. (1994); Hantos et al. (1992b,a)):

$$
\begin{aligned}
G_{r} & =\frac{1}{C_{r} \omega^{\beta_{r}}} \cos \left(\beta_{r} \frac{\pi}{2}\right) \\
H_{r} & =\frac{1}{C_{r} \omega^{\beta_{r}}} \sin \left(\beta_{r} \frac{\pi}{2}\right)
\end{aligned}
$$

The hysteresivity coefficient $\eta_{r}$ (dimensionless) is defined as (Fredberg and J (1989)):

$$
\eta_{r}=\frac{H_{r}}{G_{r}}=\tan (\beta \pi / 2)
$$

which is in fact related to the loss tangent from viscoelasticity theory and to (13) (Lai and Hyatt (2000)). This parameter characterizes the heterogeneity of the lung tissue and has been shown to vary significantly with pathology (Ionescu (2013)).

The relation between (5) and (14) stands in the frequency response. One is a theoretical model, whereas the other one is a lumped identified parametric model. Both of the express the respiratory impedance with a fractional order term.

\section{LIMITATIONS}

It is straightforward to apply airway altering/remodeling effects in the simple model representation proposed here, but limitations should be taken into account. The major errors that may occur in this study are determined by the heterogeneity of the human lung, i.e. inter-subject variability can affect the morphologic values of airway radius, thickness, length and tissue elasticity.

Another further simplification in our reasoning is considering negligible the effects from the branching angles. These angles influence the flow to change direction, may lead to an asymmetrical velocity profile, to develop a secondary flow in the daughter branches and the inner airway walls to be slightly stretched. The change in cross-sectional areas that occur from parent to daughter branches in a bifurcation causes the fluid to undergo a deceleration and may cause separation of adjoining streamlines. However, this kind of information may be more useful to study airflow dynamics in aerosol deposition models rather than in lumped impedance models.

Yet another significant limitation is the fact that structural changes are difficult to measure in a non-invasive manner and correlate with the relations described in this paper. This may be possible by performing measurements on diffusion degree in volunteers, i.e. the subject inhales pure oxygen or helium ratio and in the next 24 hours the composition of the breathing gas is non-invasively analyzed. Such studies are possible but they are costly since they require expensive gas therapy and expensive 
materials (i.e. medical gas). Ongoing efforts are expected to deliver first correlation analysis in the near future.

\section{CONCLUSION}

In this paper we provided a correlation between the structural changes occurring in the lungs and the corresponding variations in the fractional order value of an impedance model. It was discussed how variations in the wall thickness, cross-sectional area and elastic moduli in distal airways involved in respiratory process affect changes in the fractional order value. Two lumped models were discussed: i) a theoretical model derived from morphological information and ii) an identified lumped parametric model. The relationship between fractional order term and heterogeneity in the lungs has been related to changes in viscoelasticity. Our results indicate that a correlation analysis is possible for various degrees of obstruction and effects may be directly related to the identified fractional order value.

\section{REFERENCES}

Adolfsson, K., Enelund, M., and Olsson, M. (2005). On the fractional order model of viscoelasticity. Springer, Mechanics of Time- Dependent materials, 9, 15-34.

Bates, J. (2007). A recruitment model of quasi-linear power-law stress adaptation in lung tissue. Annals of Biomedical Engineering, doi:10.1007/s10439-007-92910 .

Berg, J. (2002). Biochemistry. W.H. Freeman and Company, New York.

Berg, J. (2009). Lung Mechanics - an inverse modeling approach. Cambridge Press, Cambridge.

Craiem, D. and Armentano, R. (2007). A fractional derivative model to describe arterial viscoelasticity. Biorheology, 44, 251-263.

Doehring, T., Freed, A., Carew, E., and Vesely, I. (2005). Fractional order viscoelasticity of the aortic valve cusp: an alternative to quasilinear viscoelasticity. Journal of Biomechanical Engineering, 127, 708-708.

Fredberg, J. and J, .S. (1989). On the imperfect elasticity of lung tissue. Journal of Applied Physiology, 67, 2408241.

Hantos, Z., Adamicz, A., Govaerts, E., and Daroczy (1992a). Mechanical impedances of lungs and chest wall in the cat. Journal of Applied Physiology, 73, 427-433.

Hantos, Z., Daroczy, B., Suk, B., Nagy, S., and Fredberg, J. (1992b). Input impedance and peripheral inhomogeneity of dog lungs. Journal of Applied Physiology, 72, 427-433.

Hogg, J., Chu, F., Utokaparch, S., and et al (2004). The nature of small airway obstruction in chronic obstructive pulmonary disease. New Engl J Med, 350, 2645-2653.

Ionescu, C. (2013). The human respiratory system: an analysis of the interplay between anatomy, structure, breathing and fractal dynamics. Springer, Series in BioEngineering.

Ionescu, C., Derom, E., and Keyser, R.D. (2009a). Assessment of respiratory mechanical properties with constant-phase models in healthy and copd lungs. Comp Meth and Prog in Biomed.

Ionescu, C., Hernandez, A., and Keyser, R.D. (2013). A recurrent parameter model to characterize the highfrequency range of respiratory impedance in healthy subjects. IEEE Transactions on Biomedical Circuits and Systems, 7(6), 882-892.

Ionescu, C., Segers, P., and Keyser, R.D. (2009b). Mechanical properties of the respiratory system derived from morphologic insight. IEEE Transactions on Biomedical Engineering, 56, 949-959.

Kaczka, D. and Dellaca, R. (2011). Oscillation mechanics of the respiratory system: applications to lung disease. Crit Rev Biomed Eng, 39, 337-359.

Kundur, P. (1994). Power system stability and control. Power System Engineering, Balu, Lauby (ed), New York.

Lai, S. and Hyatt, R. (2000). Effect of age on elastic moduli. J Appl Physiol, 89, 163-168.

Lande, B. and Mitzner, W. (2006). Analysis of lung parenchyma as a parametric porous medium. J Appl Physiol, 101.

Machado, J.T., Kiryakova, V., and Mainardi, F. (2013). Recent history of fractional calculus. Communications in Nonlinear Science and Numerical Simulation, 16, 1140-1153.

Magin, R. (2006). Fractional Calculus in Bioengineering. Begell House Publishers.

Oustaloup, A. (1995). La derivation non-entiere. Hermes, Paris.

Suki, B., Barabasi, A., and Lutchen, K. (1994). Lung tissue viscoelasticity: a mathematical framework and its molecular basis. J Appl Physiol, 76, 2749-2759.

Tgavalekos, N., Venegas, J., Suki, B., and Lutchen, K. (2003). Relation between structure, function and imaging in a three-dimensional model of the lung. Annals of Biomed Eng, 31, 363-373.

West, B., Barghava, V., and Goldberger, A. (1986). Beyond the principle of similitude: renormalization of the bronchial tree. J Appl Physiol, 60, 1089-1097. 